Pure and Applied Mathematics Quarterly

Volume 8, Number 4

$929-939,2012$

\title{
Local GW Invariants of Elliptic Multiple Fibers
}

\author{
Junho Lee
}

\begin{abstract}
We use simple geometric arguments to calculate the dimension zero local Gromov-Witten invariants of elliptic multiple fibers. This completes the calculation of all dimension zero GW invariants of elliptic surfaces with $p_{g}>0$.
\end{abstract}

Keywords: Local GW invariants, elliptic surfaces, spectral flow.

Let $X$ be a Kähler surface with $p_{g}>0$. By the Enriques-Kodaira classification (cf. [BHPV]), its minimal model is a $K 3$ or Abelian surface, a surface of general type or an elliptic surface. Each holomorphic 2-form $\alpha$ on $X$ defines an almost complex structure

$$
J_{\alpha}=\left(I d+J K_{\alpha}\right) J\left(I d+J K_{\alpha}\right) .
$$

Here, $J$ is the complex structure on $X$ and the endomorphism $K_{\alpha}$ of $T X$ is defined by the formula $\left\langle u, K_{\alpha} v\right\rangle=\alpha(u, v)$ where $\langle$,$\rangle is the Kähler metric. This$ $J_{\alpha}$ satisfies :

Lemma 0.1 ([L]). If $f$ is a $J_{\alpha}$-holomorphic map that represents a nontrivial $(1,1)$ class then its image lies in the support of the zero divisor $D_{\alpha}$ of $\alpha$ and $f$ is, in fact, holomorphic.

The Gromov-Witten invariant $G W_{g, n}(X, A)$ is a (virtual) count of holomorphic maps representing the class $A$. In particular, the invariant $G W_{g, n}(X, A)$ vanishes unless $A$ is a $(1,1)$ class since every holomorphic map represents $(1,1)$ class. Note 
that each canonical divisor $D$ of $X$ is a zero divisor of a holomorphic 2-form. Lemma 0.1 thus shows that the GW invariant is a sum

$$
G W_{g, n}(X, A)=\sum G W_{g, n}^{l o c}\left(D_{k}, A_{k}\right)
$$

over the connected components $D_{k}$ of the canonical divisor $D$ of local invariants that counts the contribution of maps whose image lies in $D_{k}$ (cf. [LP], [KL]). It follows that the GW invariants of minimal $K 3$ or Abelian surfaces are trivial except possibly for the trivial homology class because their canonical divisors are trivial.

The local GW invariants have a universal property. If $X$ is a minimal surface of general type with a smooth canonical divisor $D$ then the local invariants associated with $D$, and hence GW invariants, are determined by the normal bundle of $D$ - in fact, there exists a universal function of $c_{1}^{2}$ and $c_{2}$ that gives the GW invariants of $X$ (cf. Section 7 of $[\mathrm{LP}]$ ).

If $\pi: X \rightarrow C$ is a minimal elliptic surface with $p_{g}>0$, after suitable deformation, we can assume $X$ has a canonical divisor of the form

$$
\sum_{i} n_{i} F^{i}+\sum_{k}\left(m_{k}-1\right) F_{m_{k}}
$$

where $F^{i}$ is a regular fiber and $F_{m_{k}}$ is a smooth multiple fiber of multiplicity $m_{k}$ (cf. Proposition 6.1 of [LP]). In this case, the GW invariants of $X$ are sums of universal functions, and are completely determined by the multiplicities $m_{k}$ and the number

$$
c_{\pi}=\chi\left(\mathcal{O}_{X}\right)-2 \chi\left(\mathcal{O}_{C}\right)
$$

(cf. Section 6 of $[\mathrm{LP}]$ ). In particular, the generating function for the set of all dimension zero $\mathrm{GW}$ invariants of $X$ is given by

$$
G W_{X}^{0}=c_{\pi} \sum_{d>0} G W_{1}^{l o c}(F, d) t^{d}+\sum_{k} \sum_{d>0} G W_{1}^{l o c}\left(F_{m_{k}}, d\right) t_{m_{k}}^{d}
$$

where the formal variables $t$ and $t_{m_{k}}$ are for the fiber class $[F]$ and the multiple fiber classes $\left[F_{m_{k}}\right]$ respectively; these satisfy $t_{m_{k}}^{m_{k}}=t$. The local invariants in $(0.2)$ are counts of multiple covers of elliptic curves together with signs determined by the GW theory of 4-manifolds. 
Some of the generating functions in (0.2) are known. In cases of the regular fiber $F$ and the multiple fiber $F_{2}$, it was proved in Section 10 of [LP] that

$$
G W_{1}^{l o c}(F, d)=-\frac{1}{d} \sigma(d) \quad \text { and } \quad G W_{1}^{l o c}\left(F_{2}, d\right)=\frac{1}{d}\left(\sigma(d)-2 \sigma\left(\frac{d}{2}\right)\right)
$$

where $\sigma(d)=\sum_{k \mid d} k$ if $d$ is a positive integer and $\sigma(d)=0$ otherwise. In this note we use geometric arguments to obtain the terms in (0.2) associated with fibers of higher multiplicity. Our main theorem is the following formula for the local invariants $G W_{1}^{\text {loc }}\left(F_{m}, d\right)$ for $m>2$. This completes the calculation of all dimension zero GW invariants of all minimal elliptic surfaces with $p_{g}>0$.

Main Theorem. Let $m \geq 3$. Then

$$
G W_{1}^{l o c}\left(F_{m}, d\right)=\frac{1}{d}\left(\sigma(d)-m \sigma\left(\frac{d}{m}\right)\right) .
$$

The contribution of each degree $d$ cover $f$ of elliptic curve $F_{m}$ is, as a map into a 4-manifold, determined by the normal bundle $N_{m}$ of $F_{m}$. In cases of $F_{1}=F$ and $F_{2}$, the almost complex structure $J_{\alpha}$ on $X$ is generic in the sense that the linearized operator $L_{f}$ (see (1.7) below) is invertible and hence the contribution of $f$ is $(-1)^{h^{0}\left(N_{m}\right)} /|\operatorname{Aut}(f)|$ (cf. Section 10 of $[\mathrm{LP}]$ ). When $m \geq 3, J_{\alpha}$ is, in general, no longer generic. We need to perturb $J_{\alpha}$ to generic $J$. In Section 2 , using the universal property of local invariants (see (1.3) below), we choose a local model that is convenient for our calculation. In Section 3 , when $L_{f}$ is not invertible, we use a lifting property of covering space to calculate the contribution of $f$ that proves the Main Theorem. The information for dimension zero GW invariants of elliptic surfaces with $p_{g}>0$ is the same as for its Seiberg-Witten invariants. We spell out the specific connection in Remark 3.4.

Acknowledgments. I am very grateful to Thomas H. Parker for helpful discussions.

\section{Dimension Zero Genus One Local GW Invariants}

Let $X$ be a (not necessarily compact) elliptic Kähler surface with a holomorphic 2 -form $\alpha$. The 2-form $\alpha$ defines an almost complex structure $J_{\alpha}$ on $X$ by the formula (0.1). Suppose that the zero divisor $D_{\alpha}$ of $\alpha$ has a smooth reduction $D_{\alpha}=(m-1) D$ where $D$ is a regular fiber or a multiple fiber of multiplicity $m$ 
for some integer $m>1$. The adjunction formula then shows $c_{1}(T X)([D])=0$ and $c_{1}(N)=0$ where $N$ is the normal bundle of $D$. The moduli space

$$
\overline{\mathcal{M}}_{1}^{\alpha}(X, d[D])
$$

of stable $J_{\alpha}$-holomorphic maps from curves of genus one representing the class $d[D](d \neq 0)$ carries a (virtual) fundamental class

$$
\left[\overline{\mathcal{M}}_{1}^{\alpha}(X, d[D])\right]^{v i r}
$$

that is defined by the GW theory of 4 -manifolds (cf. Section 4 of $[\mathrm{LP}]$ ). This (virtual) fundamental class (1.2) has dimension zero since $c_{1}(T X)([D])=0$. The dimension zero genus one local Gromov-Witten invariant of $X$ associated with the zero divisor $D_{\alpha}$ is then

$$
G W_{1}^{l o c}\left(X, D_{\alpha}, d\right):=\left[\overline{\mathcal{M}}_{1}^{\alpha}(X, d[D])\right]^{v i r} .
$$

This local GW invariant has the following universal property. Let $X^{\prime}$ be another elliptic Kähler surface with a holomorphic 2-form $\alpha^{\prime}$ whose zero divisor $D_{\alpha^{\prime}}=$ $\left(m^{\prime}-1\right) D^{\prime}$ where $D^{\prime}$ is a regular fiber or a multiple fiber of multiplicity $m^{\prime}$. Let $N^{\prime}$ be the normal bundle of $D^{\prime}$. If $m=m^{\prime}$ and $h^{0}(N)=h^{0}\left(N^{\prime}\right)$ then

$$
G W_{1}^{l o c}\left(X, D_{\alpha}, d\right)=G W_{1}^{l o c}\left(X^{\prime}, D_{\alpha^{\prime}}, d\right)
$$

(cf. Section 6 of $[\mathrm{LP}])$. We set

$$
G W_{1}^{\text {loc }}\left(X, D_{\alpha}, d\right)= \begin{cases}G W_{1}^{l o c}((m-1) F, d) & \text { if } D \text { is a regular fiber } \\ G W_{1}^{l o c}\left(F_{m}, d\right) & \text { if } D \text { is a } m \text {-multiple fiber }\end{cases}
$$

It was proved in Example 4.4 of $[\mathrm{LP}]$ that

$$
G W_{1}^{l o c}(m F, d)=m G W_{1}^{l o c}(F, d) .
$$

As given in (0.2), all dimension zero GW invariants of minimal elliptic surfaces with $p_{g}>0$ are sums of local invariants in (1.4).

In the below, we will give a precise description on the (virtual) fundamental class (1.2) which will be used for our calculation in Section 3. The point in the moduli space (1.1) is an equivalence class $[f, C]$ of stable maps $(f, C)$ where two stable maps $(f, C)$ and $\left(f^{\prime}, C^{\prime}\right)$ are equivalent if there is a biholomorphic map $\sigma: C \rightarrow C^{\prime}$ with $f^{\prime} \circ \sigma=f$. By Lemma 0.1 , if $d \neq 0$ every representative $(f, C)$ of $[f, C]$ is a holomorphic $d$-fold covering map from $C$ to $D$. Thus, if $D$ is given by 
a lattice $\Lambda$ in the complex plane then $[f, C]$ is determined by an index $d$ sublattice of $\Lambda$. In particular, the moduli space (1.1) consists of $\sigma(d)$ points.

On the other hand, since the (virtual) fundamental class (1.2) is defined by the GW theory of 4-manifolds, as described in Section 3 of [IP], it is a finite sum

$$
\left[\overline{\mathcal{M}}_{1}^{\alpha}(X, d[D])\right]^{v i r}=\sum c([f, C])
$$

over $[f, C] \in \overline{\mathcal{M}}_{1}^{\alpha}(X, d[D])$ of the contributions $c([f, C])$ that are defined as follows. Choose a $p \in D$ and a small disk $B$ in $X$ with $B \cap D=\{p\}$ and, once and for all, fix a map $(f, C, x)$ with $f(x)=p$ such that $(f, C)$ represents $[f, C]$. Then for a generic almost complex structure $J$ on $X$ that is sufficiently close to $J_{\alpha}$ and tamed by the Kähler form on $X$, there are finitely many $J$-holomorphic maps $\left(f_{i}, C_{i}, x_{i}\right)$ from smooth genus one curves with one marked point such that (i) $f_{i}\left(x_{i}\right) \in B$ (ii) each $\left(f_{i}, C_{i}, x_{i}\right)$ is $C^{0}$-close to $(f, C, x)$ (in a suitable space of maps) and (iii) the index zero operator

$$
L_{f_{i}}: \Omega^{0}\left(f_{i}^{*} N_{i}\right) \rightarrow \Omega^{0,1}\left(f_{i}^{*} N_{i}\right)
$$

has trivial kernel (or equivalently $L_{f_{i}}$ is invertible) where the operator $L_{f_{i}}$ is obtained by linearizing $J$-holomorphic map equation (see Remark 1.1 below) and restricting to the normal bundle $N_{i}$ of the image of $f_{i}$. Denote by

$$
\mathcal{M}_{(f, C, x), B, J}
$$

the set of such $J$-holomorphic maps $\left(f_{i}, C_{i}, x_{i}\right)$. Notice that for each $\left(f_{i}, C_{i}, x_{i}\right)$ the preimage $f_{i}^{-1}(B)$ consists of $d=|\operatorname{Aut}(f)|$ distinct points $x_{i j}$. Since the automorphism group of $C_{i}$ acts transitively, for each $x_{i j}$ there exists an automorphism $\sigma_{j}$ of $C_{i}$ with $\sigma_{j}\left(x_{i}\right)=x_{i j}$ such that $\left(f_{i} \circ \sigma_{j}, C_{i}, x_{i}\right)$ is also contained in the set $\mathcal{M}_{(f, C, x), B, J}$. The contribution $c([f, C])$ is thus the (weighted) sum

$$
c([f, C])=\frac{1}{d} \sum(-1)^{S F\left(L_{f_{i}}\right)}
$$

over $f_{i}$ in $\mathcal{M}_{(f, C, x), B, J}$ where the sign of each $f_{i}$ is given by the mod 2 spectral flow $S F\left(L_{f_{i}}\right)$ of the invertible operator $L_{f_{i}}$. In particular, $S F\left(L_{f_{i}}\right)=0$ if $L_{f_{i}}$ is complex linear, namely $J$-linear.

Remark 1.1. The operator $D_{f_{i}}: \Omega^{0}\left(f_{i}^{*} T X\right) \rightarrow \Omega^{0,1}\left(f_{i}^{*} T X\right)$ obtained by linearizing $J$-holomorphic map equation at $f_{i}$ is given by

$$
D_{f_{i}}(\xi)(v)=\nabla_{v} \xi+J \nabla_{j v} \xi+\frac{1}{2}\left[\left(\nabla_{\xi} J\right)\left(d f_{i}(j v)\right)-J\left(\nabla_{\xi} J\right)(v)\right]
$$


where $\xi \in \Omega^{0}\left(f_{i}^{*} T X\right), v \in T C_{i}$ and $j$ is the complex structure on $C_{i}$. Here $\nabla$ is the pull-back connection on $f_{i}^{*} T X$ of the Levi-Civita connection of the metric on $X$ that is defined by the Käler form and $J$ (cf. Lemma 6.3 of [RT]).

\section{LOCAL MODEL}

Once and for all, fix an integer $m \geq 2$ and let $D$ denote the elliptic curve given as the complex plane (with coordinate $z$ ) modulo the lattice $\mathbb{Z}+(m i) \mathbb{Z}$. Then $S=D \times \mathbb{C}$ has an automorphism $\varphi$ of order $m$ defined by

$$
\varphi(z, w)=\left(z+i, e^{2 \pi i / m} \cdot w\right)
$$

such that all powers $\varphi^{i}$ are fixed-point free where $w$ is a coordinate on $\mathbb{C}$. Let $S_{m}$ be the quotient of $S$ by the group $\left\{\varphi^{i}\right\}$ and $q: S \rightarrow S_{m}$ the quotient map. The map $S \rightarrow \mathbb{C}:(z, w) \rightarrow w^{m}$ then factors through $S_{m}$ to give an elliptic fibration $S_{m} \rightarrow \mathbb{C}$ whose central fiber is a $m$-multiple fiber $D_{m}$ given by the lattice $\mathbb{Z}+i \mathbb{Z}$ with torsion normal bundle $N_{m}$ of order $m$ :

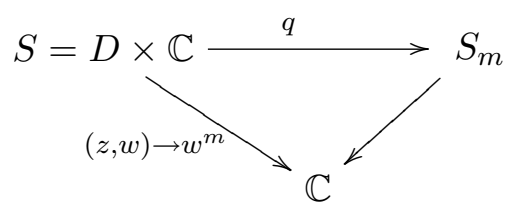

The following simple observation is a key fact for our subsequent discussions. Let $f: C \rightarrow D_{m}$ be a holomorphic map of degree $d$ from an elliptic curve $C$ that is given by a sublattice of $\mathbb{Z}+i \mathbb{Z}$ of the form

$$
a \mathbb{Z}+(b i+k) \mathbb{Z} \quad \text { with } \quad d=a b, \quad 0 \leq k \leq a-1 .
$$

Write $D \times\{0\} \subset S$ simply as $D$.

Lemma 2.1. Let $D, N_{m}$ and $f: C \rightarrow D_{m}$ be as above. Then,

$$
f \text { factors through } D \quad \Longleftrightarrow a \mid \frac{d}{m} \quad \Longleftrightarrow \quad f^{*} N_{m}=\mathcal{O}_{C}
$$

Proof. $f$ factors through $D \Longleftrightarrow a \mathbb{Z}+(b i+k) \mathbb{Z}$ is a sublattice of $\mathbb{Z}+(m i) \mathbb{Z} \Longleftrightarrow$ $m|b \Longleftrightarrow a| \frac{d}{m}$. This shows the first assertion. Observe that for the restriction $\operatorname{map} g_{m}=q_{\mid D}: D \rightarrow D_{m}$

$$
g_{m}^{*}\left(N_{m}\right)=g_{m}^{*}\left(\left[D_{m}\right]_{\mid D_{m}}\right)=q^{*}\left(\left[D_{m}\right]\right)_{\mid D}=\left[q^{*} D_{m}\right]_{\mid D}=[D]_{\mid D}=\mathcal{O}_{D}
$$


where $\left[D_{m}\right]$ is the line bundle associated to the divisor $D_{m}, N_{m}=\left[D_{m}\right]_{\mid D_{m}}$ by adjunction, the pullback divisor $q^{*} D_{m}=D$ and again by adjunction $[D]_{\mid D}$ is the normal bundle of $D$ that is trivial. Write as $N_{m}=\mathcal{O}_{D_{m}}(p-q)$ where

$$
\int_{q}^{p} d z=\frac{k_{1}}{m}+i \frac{k_{2}}{m} \quad \text { for some } \quad 0 \leq k_{1}, k_{2} \leq m-1 .
$$

Then, by (2.1) and the Abel's Theorem, $g_{m}^{*} N_{m}=\mathcal{O}_{D}\left(\sum_{j}\left(p_{j}-q_{j}\right)\right)$ for some $p_{j}, q_{j}$ such that

$$
\sum_{j} \int_{q_{j}}^{p_{j}} d z=k_{1}+i k_{2} \equiv 0 \quad \bmod \quad \mathbb{Z}+(m i) \mathbb{Z} .
$$

Consequently, $k_{2}=0$ and $\operatorname{gcd}\left(m, k_{1}\right)=1$ since $N_{m}$ is torsion of order $m$. Now, again by the Abel's Theorem, $f^{*} N_{m}=\mathcal{O}_{C}\left(\sum_{\ell}\left(t_{\ell}-s_{\ell}\right)\right)$ for some $s_{\ell}, t_{\ell}$ such that

$$
\sum_{\ell} \int_{s_{\ell}}^{t_{\ell}} d z=\frac{d k_{1}}{m} \equiv 0 \quad \bmod \quad a \mathbb{Z}+(b i+k) \mathbb{Z} \quad \Longleftrightarrow \quad f^{*} N_{m}=\mathcal{O}_{C}
$$

Therefore, $a\left|\frac{d}{m} \Longleftrightarrow m\right| b \Longleftrightarrow a \mid \frac{d k_{1}}{m} \Longleftrightarrow f^{*} N_{m}=\mathcal{O}_{C}$. This shows the second assertion.

Remark 2.2. Since $q: S \rightarrow S_{m}$ is a covering map, Lemma 2.1 shows $f: C \rightarrow$ $D_{m} \subset S_{m}$ lifts to $\tilde{f}: C \rightarrow D \subset S$ if and only if $f^{*} N_{m}=\mathcal{O}_{C}$. On the other hand, the Kähler form $-\frac{i}{2}(d z \wedge d \bar{z}+d w \wedge d \bar{w})$ on $\mathbb{C}^{2}$ descends to a Kähler form $\tilde{\omega}$ on $S$ that is $\varphi$-invariant, so $\tilde{\omega}$ also descends to a Kähler form $\omega$ on $S_{m}$ such that $q^{*} \omega=\tilde{\omega}$.

\section{Calculation}

Let $q:(S, D) \rightarrow\left(S_{m}, D_{m}\right)$ be as in Section 2. Fix a holomorphic 2-from

$$
\alpha=w^{m-1} d w \wedge d z
$$

on $S$ whose zero divisor is $(m-1) D$ and let $J_{\alpha}$ denote the almost complex structure on $S$ defined by the formula (0.1). The 2 -form $\alpha$ is $\varphi$-invariant, so it descends to a holomorphic 2-form $\alpha_{m}$ on $S_{m}$ whose zero divisor is $(m-1) D_{m}$. We denote by $J_{m}=J_{\alpha_{m}}$ the almost complex structure on $S_{m}$ defined by the 2 -form $\alpha_{m}$. Since $D_{m}$ is a multiple fiber of multiplicity $m$,

$$
G W_{1}^{l o c}\left(F_{m}, d\right)=\left[\overline{\mathcal{M}}_{1}^{\alpha_{m}}\left(S_{m}, d\left[D_{m}\right]\right)\right]^{\text {vir }}
$$


where the right-hand side is given by the sum of contributions as in (1.6). In order to calculate them, we decompose the moduli space $\overline{\mathcal{M}}_{1}^{\alpha_{m}}\left(S_{m}, d\left[D_{m}\right]\right)$ as a disjoint union

$$
\overline{\mathcal{M}}_{1}^{\alpha_{m}}\left(S_{m}, d\left[D_{m}\right]\right)=\mathcal{M}_{m, d}^{+} \amalg \mathcal{M}_{m, d}^{-}
$$

where

$$
\mathcal{M}_{m, d}^{+}=\left\{[f, C]: h^{0}\left(f^{*} N_{m}\right)=0\right\} \text { and } \mathcal{M}_{m, d}^{-}=\left\{[f, C]: h^{0}\left(f^{*} N_{m}\right)=1\right\} .
$$

It then follows from Lemma 2.1 that

$$
\# \mathcal{M}_{m, d}^{+}=\sigma(d)-\sigma\left(\frac{d}{m}\right) \quad \text { and } \quad \# \mathcal{M}_{m, d}^{-}=\sigma\left(\frac{d}{m}\right)
$$

where $\# A$ is the cardinality of a set $A$.

We first calculate the contribution $c([f, C])$ of $[f, C]$ in $\mathcal{M}_{m, d}^{+}$. In the below, we always assume $m \geq 3$ and $m \mid d$.

Lemma 3.1. If $[f, C] \in \mathcal{M}_{m, d}^{+}$then $c([f, C])=\frac{1}{d}$.

Proof. The linearized operator $L_{f}$ has the form $L_{f}=\bar{\partial}_{f}+R_{m}$ where $\bar{\partial}_{f}$ is the usual $\bar{\partial}$-operator on $f^{*} N_{m}$ and the zeroth order term $R_{m}$ is given by

$$
R_{m}(\xi)=-\nabla_{\xi} K_{\alpha_{m}} \circ J_{\alpha_{m}} \circ d f \quad \text { for } \quad \xi \in \Omega^{0}\left(f^{*} N_{m}\right)
$$

(cf. Section 8 of [LP]). But, $R_{m} \equiv 0$ since $\alpha_{m}$ (and hence $K_{\alpha_{m}}$ ) vanishes of order $m-1 \geq 2$ along $D_{m}$. Consequently, dim ker $L_{f}=2 h^{0}\left(f^{*} N_{m}\right)=0$, so $L_{f}$ is invertible with $S F\left(L_{f}\right)=0$. Now, the proof follows from the fact $f: C \rightarrow D_{m}$ has degree $d$.

Let $[f, C] \in \mathcal{M}_{m, d}^{-}$. The proof of Lemma 3.1 shows $L_{f}=\bar{\partial}_{f}$ is not invertible. In this case, we will uses the $m$-fold covering map $q: S \rightarrow S_{m}$ to calculate the contribution $c([f, C])$. Observe that by Lemma 2.1 the map

$$
\mathcal{M}_{m, d}^{-} \rightarrow \overline{\mathcal{M}}_{1}^{\alpha}\left(S, \frac{d}{m}[D]\right) \quad \text { defined by } \quad[f, C] \rightarrow[\tilde{f}, C]
$$

is one-to-one and onto where $\tilde{f}$ is a lift of $f$.

Lemma 3.2. If $[f, C] \in \mathcal{M}_{m, d}^{-}$then $c([f, C])=\frac{1}{m} c([\tilde{f}, C])$.

Proof. Let $B=\{0\} \times \Delta \subset S$ where $\Delta$ is a small disk around 0 in $\mathbb{C}$ and $B_{m}=q(B)$ and fix a map $(f, C, x)$ with $f(x) \in B_{m}$ such that $(f, C)$ represents $[f, C]$. Since the restriction map $q_{\mid B}: B \rightarrow B_{m}$ is one-to-one, Lemma 2.1 shows 
that $(f, C, x)$ uniquely lifts to a $J_{\alpha}$-holomorphic map $(\tilde{f}, C, x)$ with $\tilde{f}(x) \in B$ such that $(\tilde{f}, C)$ represents $[\tilde{f}, C]$ in $\overline{\mathcal{M}}_{1}^{\alpha}\left(S, \frac{d}{m}[D]\right)$.

Let $\omega$ and $\tilde{\omega}$ be the Kähler forms as in Remark 2.2 and choose a generic $\omega$ tamed almost complex structure $J$ on $S_{m}$ that is close to $J_{m}$. Then, we have

- $J$ lifts to an $\tilde{\omega}$-tamed almost complex structure $\tilde{J}$ on $S$ close to $J_{\alpha}$ such that $d q \circ \tilde{J}=J \circ d q$,

- each $f_{i}$ in $\mathcal{M}_{(f, C, x), B_{m}, J}$ is homotopic to $f$ since $f_{i}$ is $C^{0}$-close to $f$, so $\left(f_{i}, C_{i}, x_{i}\right)$ also uniquely lifts to $\tilde{J}$-holomorphic maps $\left(\tilde{f}_{i}, C_{i}, x_{i}\right)$ with $\tilde{f}\left(x_{i}\right) \in B$ such that $\left(\tilde{f}_{i}, C_{i}, x_{i}\right)$ is $C^{0}$-close to $(\tilde{f}, C, x)$.

The pair $(\omega, J)$ defines a metric $g$ on $S_{m}$ whose lift $\tilde{g}=q^{*} g$ is the same metric defined by the pair $(\tilde{\omega}, \tilde{J})$. Let $\nabla$ and $\tilde{\nabla}$ respectively denote the pull-back connections on $f_{i}^{*} T S_{m}$ and $\tilde{f}_{i}^{*} T S$ of the Levi-Civita connection of $g$ and $\tilde{g}$. The differential $d q$ then induces a bundle isomorphism $d q: \tilde{f}_{i}^{*} T S \rightarrow \tilde{f}_{i}^{*} q^{*} T S_{m}=$ $f_{i}^{*} T S_{m}$ such that $d q \circ \tilde{\nabla}=\nabla \circ d q$ (see [W] page 138) and hence by the formula (1.8) we have

$$
d q \circ D_{\tilde{f}_{i}}=D_{f_{i}} \circ d q
$$

The differential $d q$ also induces a bundle isomorphism $d q_{i}: \tilde{f}_{i}^{*} \tilde{N}_{i} \rightarrow f_{i}^{*} N_{i}$ and restricting the equation (3.2) to $\tilde{f}_{i}^{*} \tilde{N}_{i}$ and $f_{i}^{*} N_{i}$ gives

$$
d q_{i} \circ L_{\tilde{f}_{i}}=L_{f_{i}} \circ d q_{i}
$$

where $\tilde{N}_{i}$ and $N_{i}$ are normal bundles of $\operatorname{Im}\left(\tilde{f}_{i}\right)$ and $\operatorname{Im}\left(f_{i}\right)$ respectively. Therefore, $L_{\tilde{f}_{i}}$ is also invertible and hence there is one-to-one correspondence

$$
\mathcal{M}_{(f, C, x), B_{m}, J} \rightarrow \mathcal{M}_{(\tilde{f}, C, x), B, \tilde{J}} \quad \text { given by } \quad\left(f_{i}, C_{i}, x_{i}\right) \rightarrow\left(\tilde{f}_{i}, C_{i}, x_{i}\right) .
$$

Let $\tilde{L}_{t}$ be a path of first order elliptic operators from an invertible $\tilde{J}_{\text {-linear }}$ operator $\tilde{L}_{0}$ to $\tilde{L}_{1}=L_{\tilde{f}_{i}}$ with all $\tilde{L}_{t}$ invertible except at finitely many $t_{k}$. Then, $d q_{i} \circ \tilde{L}_{t} \circ\left(d q_{i}\right)^{-1}$ is also a path from invertible $J$-linear operator to $L_{f_{i}}$ such that $S F\left(L_{\tilde{f}_{i}}\right) \equiv \sum_{k} \operatorname{dim} \operatorname{ker} \tilde{L}_{t_{k}}=\sum_{k} \operatorname{dim} \operatorname{ker} d q_{i} \circ \tilde{L}_{t_{k}} \circ\left(d q_{i}\right)^{-1} \equiv S F\left(L_{f_{i}}\right) \quad(\bmod 2)$. Now, noting $\operatorname{deg}(f)=d$ and $\operatorname{deg}(\tilde{f})=\frac{d}{m}$, we have

$$
c([f, C])=\frac{1}{d} \sum_{f_{i}}(-1)^{S F\left(L_{f_{i}}\right)}=\frac{1}{d} \sum_{\tilde{f}_{i}}(-1)^{S F\left(L_{\tilde{f}_{i}}\right)}=\frac{1}{m} c([\tilde{f}, C]) .
$$


We are now ready to prove the Main Theorem in the introduction.

Proof of the Main Theorem : It follows from Lemma 3.1, Lemma 3.2 and (3.1) that

$$
\begin{aligned}
G W_{1}^{l o c}\left(F_{m}, d\right) & =\sum_{[f, C] \in \mathcal{M}_{m, d}^{+}} c([f, C])+\sum_{[f, C] \in \mathcal{M}_{m, d}^{-}} c([f, C]) \\
& =\frac{1}{d}\left(\sigma(d)-\sigma\left(\frac{d}{m}\right)\right)+\frac{1}{m}\left[\overline{\mathcal{M}}_{1}^{\alpha}\left(S, \frac{d}{m}[D]\right)\right]^{v i r} .
\end{aligned}
$$

Since the 2-form $\alpha$ on $S$ has the zero divisor $(m-1) D$, so by $(1.5)$ and $(0.3)$ we have

$$
\left[\overline{\mathcal{M}}_{1}^{\alpha}\left(S, \frac{d}{m}[D]\right)\right]^{v i r}=G W_{1}^{l o c}\left((m-1) F, \frac{d}{m}\right)=-(m-1) \frac{m}{d} \sigma\left(\frac{d}{m}\right) .
$$

Now, the proof follows from (3.3) and (3.4).

Remark 3.3. One can also use the above argument to compute $G W_{1}^{\text {loc }}\left(F_{2}, d\right)$, replacing the "Taubes type" argument used in [LP]. Specifically, for each $f$ in $\overline{\mathcal{M}}_{1}^{\alpha_{2}}\left(S_{2}, d\left[D_{2}\right]\right)$ the linearized operator $L_{f}$ is invertible with $S F\left(L_{f}\right) \equiv h^{0}\left(f^{*} N_{2}\right)$ $(\bmod 2)$ (cf. Proposition 9.2 of $[\mathrm{LP}])$. Thus, by (3.1) we have

$$
\begin{aligned}
G W_{1}^{l o c}\left(F_{2}, d\right) & =\left[\overline{\mathcal{M}}_{1}^{\alpha_{2}}\left(S_{2}, d\left[D_{2}\right]\right)\right]^{\text {vir }}=\frac{1}{d}\left(\sigma(d)-\sigma\left(\frac{d}{2}\right)\right)-\frac{1}{d} \sigma\left(\frac{d}{2}\right) \\
& =\frac{1}{d}\left(\sigma(d)-2 \sigma\left(\frac{d}{2}\right)\right) .
\end{aligned}
$$

Remark 3.4. Ionel and Parker [IP] showed how GW invariants for the class $A$ of a symplectic 4-manifold $X$ are related with the Taubes' Gromov invariants $G r_{X}(A)[\mathrm{T}]$ that count embedded (not necessarily connected) curves in $X$ representing the class $A$. They used a particular function $F(t)$ that satisfies

$$
\prod_{d} F\left(t^{d}\right)^{-\frac{1}{d} \sigma(d)}=(1-t)
$$

to relate Taubes' counting of multiple covers of embedded tori with the dimension zero genus one GW invariants. Let $X$ be a minimal elliptic surface with $p_{g}>0$. In this case, any GW invariant constrained to pass through generic points vanishes (cf. Corollary 3.4 of [LP]). So, by (0.2), (0.3) and the Main Theorem, the relation 
between two set of invariants (Theorem 4.5 of [IP]) yields

$$
\begin{aligned}
\sum_{A} G r_{X}(A) t_{A} & =\prod_{d, k} F\left(t^{d}\right)^{c_{\pi} G W_{1}^{l o c}(F, d)} F\left(t_{m_{k}}^{d}\right)^{G W_{1}^{l o c}\left(F_{m_{k}}, d\right)} \\
& =(1-t)^{c_{\pi}} \prod_{k}\left(1+t_{m_{k}}+\cdots+t_{m_{k}}^{m_{k}-1}\right) .
\end{aligned}
$$

This also gives the well-known Seiberg-Witten invariants $S W$ of $X$ (cf. [FM], [B], [FS]) due to the famous Taubes' theorem $S W=G r$.

\section{REFERENCES}

[B] R. Brussee, The canonical class and the $C^{\infty}$ properties of Khler surfaces, New York J. Math. 2 (1996), 103-146.

[BHPV] W. Barth, K. Hulek, C. Peters, and A. Van de Ven, Compact complex surfaces, second ed., Springer-Verlag, Berlin Heidelberg, 2004.

[FM] R. Friedman and J. Morgan, Obstruction bundles, semiregularity, and Seiberg-Witten invariants, Comm. Anal. Geom. 7 (1999), no. 3, 451-495.

[FS] R. Fintushel and R. Stern, Rational blowdowns of smooth 4-manifolds, J. Diff. Geom. 46 (1997), 181-235.

[IP] E. Ionel and T.H. Parker, The Gromov invariants of Ruan-Tian and Taubes, Math. Res. Lett. 4 (1997), no. 4, 521-532.

[KL] Y-H. Kiem and J. Li, Low degree GW invariants of spin surfaces, Pure Appl. Math. Q. 7 (2011), no. 4, 1449-1476.

[L] J. Lee, Family Gromov-Witten Invariants for Kähler Surfaces, Duke Math. J. 123 (2004), no. 1, 209-233.

[LP] J. Lee and T.H. Parker, A Structure Theorem for the Gromov-Witten Invariants of Kähler Surfaces, J. Diff. Geom. 77 (2007), 483-513.

[RT] Y. Ruan and G. Tian, A mathematical theory of cohomology, J. Differential Geom. 42 (1995), 259-367.

[T] C. Taubes, Seiberg Witten and Gromov invariants for symplectic 4-manifolds, Edited by Richard Wentworth. First International Press Lecture Series, 2. International Press, Somerville, MA, 2000.

[W] G. Walschap, Metric structures in differential geometry, Graduate Texts in Mathematics, 224. Springer-Verlag, New York, 2004.

Junho Lee

University of Central Florida

Orlando, FL 32816, USA

E-mail: junho.lee@ucf.edu 